\title{
CONSERVACIÓN [CRÍTICA] SOCIAL EN ARQUEOLOGÍA
}

\author{
SOCIAL [CRITICAL] CONSERVATION IN ARCHAEOLOGY
}

\author{
Ana Pastor Pérez y Margarita Díaz-Andreu ${ }^{1,2,3}$
}

\begin{abstract}
Este texto busca generar nuevos diálogos entre la crítica social que se está realizando en patrimonio y lo que llamamos una conservación social, ciñendo nuestra reflexión al mundo de la arqueología. Se intentará trazar un puente reflexivo entre las corrientes sociales de la arqueología pública y comunitaria y la conservación. Para ello exploramos los cambios que se han producido en el marco teórico de la gestión y valores del patrimonio en los últimos años y su proyección en la conservación de bienes culturales arqueológicos. El texto también analiza el debate que se genera en torno a conservación, valores y obsolescencia, proponiendo una conservación que contribuya a la [de]cosificación y des-elitización del patrimonio. Por último, proponemos algunas reflexiones finales que pincelan el futuro rol de la conservación en la arqueología de la contemporaneidad.

Palabras claves: conservación crítica, patrimonio crítico, arqueología, valores del patrimonio, gestión de patrimonio, arqueología pública, arqueología comunitaria.
\end{abstract}

This text aims to generate new dialogues between the current social critique of heritage and what we describe as 'social conservation', focusing our reflection on the archaeological field. We will attempt to draw a reflective bridge between the social trends of public and community archaeology and conservation. We will explore the changes that have occurred in the theoretical framework of heritage management and values in recent years, together with their projection in the conservation of archaeological cultural assets. We will analyze the debate generated around conservation, values, and obsolescence, advancing a conservation proposal that contributes to the [de]reification and de-elitization of heritage. Lastly, we will offer some final reflections that outline the future role of conservation in the archaeology of contemporaneity.

Key words: Critical conservation, critical heritage, archaeology, heritage values, heritage management, public archaeology, community archaeology.

\section{Patrimonio, Sociedad y Conservación Patrimonial}

La gestión del patrimonio como tal es una disciplina asociada a la creación del Estado moderno llevada a cabo, hoy en día, a partir de entes administrativos que tienen como misión proteger, conservar o difundir el patrimonio cultural. Se trata, además, de un campo académico que cubre un amplio rango de aspectos, lo que ha generado la existencia de profesionales que desde hace unas pocas décadas se definen a sí mismos como gestores culturales (Querol 2011). El trabajo que exponemos bajo estas líneas nace de una reflexión teórica sobre la conservación de bienes culturales arqueológicos, que pretende entrelazar, desde un punto de vistas transdisciplinar y reflexivo, distintas aproximaciones en torno al trinomio conservación-arqueología-sociedad. Se trata de un texto que invita al lector a cuestionar nuevas formas de abordar la conservación de bienes arqueológicos y cómo adaptarlas a la preservación de las distintas narrativas que pueden converger en un espacio, y que se examinan habitualmente desde la teoría arqueológica y patrimonial.

La participación de la sociedad en el ciclo de gestión del patrimonio, así como el rol que juegan los gestores en su interacción, es un tema considerablemente explorado y cuestionado en los últimos tiempos (Alonso-González et al. 2018; Molina Neira 2019; Quintero Morón y Sánchez Carretero 2017). Según la experta María Ángeles Querol, una característica propia de todo bien cultural es que su

\footnotetext{
Departament de Història i Arqueología, Universitat de Barcelona, Barcelona,España. a.pastor.restaura@ gmail.com; m.diaz-andreu@ub.edu Grup d'Arqueologia Pública i Patrimoni, Universitat de Barcelona, España.

ICREA - Institució Catalana de Recerca i Estudis Avançats, Barcelona, España.
}

Recibido: abril 2020. Aceptado: octubre 2020.

http://dx.doi.org/10.4067/S0717-73562021005002602. Publicado en línea: 27-diciembre-2021. 
razón de ser es social, es decir, que sus valores pueden ser disfrutados por toda la sociedad, o dejan de tener sentido como patrimonio cultural (Querol 2010:13). Habitualmente son los poderes públicos y los expertos y expertas, en muchos casos, arqueólogos/as, los encargados de gestionar el patrimonio y generar esas interacciones sociales, que suelen ser de arriba-abajo, en vertical, aplicadas desde los poderes públicos a la ciudadanía (Fiske 1992). Citando al antropólogo experto en patrimonio Llorenç Prats:

no se necesita más que la voluntad del vecindario y el apoyo de un técnico o una técnica en gestión patrimonial para convertir aquello que se decida patrimonializar en un instrumento de encuentro y debate de personas y grupos sociales, de reflexión y construcción de identidades y todo tipo de proyectos colectivos (Prats 2012:75).

Prats indica que cuando existe un deseo de cuidado hacia un elemento patrimonial, este se convierte en un potencial instrumento de encuentro, de reflexión y de debate entre diferentes agentes sociales, incluida la población local, el vecindario. El objeto patrimonial se convierte así en un creador de identidad, y esta voluntad de conservar su potencial social ha de ser canalizada por los técnicos de gestión a través de la organización de encuentros entre expertos y sociedad, siendo estos un proceso clave en la gestión patrimonial. Pero yendo más a lo concreto ¿cuál sería entonces el rol de ese "técnico de patrimonio al servicio del vecindario" que propone Prats?

Han pasado más de 40 años desde que el geógrafo norteamericano y experto en patrimonio David Lowenthal comenzase a cuestionar qué era y qué no era patrimonio (1998, 2014 [1985]). Recordamos un fragmento de su obra The Heritage Crusade and the Spoils of History (1998):

De repente, el patrimonio está en todas partes -en las noticias, en las películas, en el mercado-, en todo, desde las galaxias hasta los genes. Es el principal foco de patriotismo y un atractivo turístico de primer orden. Uno apenas puede moverse sin toparse con un sitio patrimonial. Cada legado es apreciado. Desde las raíces étnicas hasta los parques temáticos de la historia, desde Hollywood hasta el
Holocausto, el mundo entero está ocupado alabando -o lamentando- algún pasado, ya sea real o ficticio (Lowenthal 1998:xiii) (traducción de las autoras).

Para Lowenthal, al igual que para Prats, el patrimonio enraíza también con la cuestión identitaria, indicando por otra parte que todo es patrimonio, o es patrimonializable. Años más tarde Laurajane Smith crearía el concepto de Discurso Patrimonial Autorizado (Authorized Heritage Discourse) empleado como parte del aparato de poder; aquel que decide qué es y no es el patrimonio, en una gestión de arriba-abajo (Smith 2006). Las críticas a esta gestión arribaabajo han conducido a una paulatina búsqueda de democratización de los procesos patrimoniales. Rodney Harrison describía de forma muy personal el porqué de su decisión de redactar un libro que conjugase, por una parte, la historia de la disciplina, y por otra su relación con la filosofía posmoderna para entender el rol que juegan los expertos, que a su vez también son habitantes de un lugar:

Me estaba sintiendo cada vez menos cómodo con el espacio que parecía estarse creando entre los profesionales de patrimonio y los no expertos. En mis tareas profesionales enfocadas a los aspectos técnicos y científicos del patrimonio, parecía complicado incluir los puntos de vista de los agentes implicados y de los miembros de la comunidad que vivían cada día con el patrimonio hacia el que yo estaba comprometido a dar un consejo profesional (Harrison 2013a:xi) (traducción de las autoras).

El autor expresaba aquí un sentimiento con el que muchos profesionales se sentirán identificados: el rol que juegan los expertos como bisagra entre los entes de gestión y la sociedad y sus tradiciones. En esta última década, la preocupación de los expertos acerca de que no se estén reflejando las necesidades reales que se generan entre los agentes sociales y los gestores de patrimonio está generando numerosos debates y sesiones en congresos, algunos de los cuales han dado lugar a publicaciones (Apaydin 2020; Auclair y Fairclough 2015; Ireland y Schofield 2015; Rivolta et al. 2014; Sørensen y Carman 2009). Estos trabajos ilustran la necesidad dentro de la profesión de adentrarnos en una reflexión acerca de cómo 
democratizar las acciones de crear y conservar el patrimonio que recaen en los expertos, y entender la gestión del patrimonio de forma dialógica (Harrison 2013:41), es decir, como resultado de diálogos igualitarios (Londoño 2014; Schofield 2009; Smith e Iversen 2014; Waterton 2015; Waterton y Watson 2013).

Podríamos argumentar, sin embargo, que todos estos debates han evolucionado hacia un "Discurso Académico Patrimonial Autorizado", como ya vaticinaba la propia Laurajane Smith cuando indicaba que fuera de la academia los arqueólogos ejercemos muchas veces como gestores del patrimonio cultural, y esta gestión implica un rol político, especialmente cuando trata sobre temas de identidad (Smith 2004:110). Un buen reflejo de ello lo encontramos en trabajos de impacto donde se tacha a los integrantes de las denominadas corrientes críticas de patrimonio de mantener conductas paternalistas o extractivistas hacia la sociedad, indicando a la vez que son los mismos académicos los que deben "educar a la sociedad" para liberarla de un patrimonio mercantilizado (GonzálezRuibal et al. 2018:511; Pastor Pérez y Ruiz Martínez 2020). En este sentido, reconocemos que nosotras mismas somos partícipes de este discurso académico autorizado, pero a través de este trabajo, nos gustaría pensar que la conservación arqueológica social, y en el futuro, su desarrollo metodológico, podrá estar al servicio de un aprendizaje que no trate únicamente de "defender los cotos de las disciplinas con sus deslindes epistémicos, sociales y lingüísticos” (Haber 2019:139).

\section{El rol social de la arqueología contemporánea: de la excavación a la conservación arqueológica}

Para hablar de conservación crítica es necesario entender el rol social defendido desde la arqueología crítica (y social) hoy en día. Sin entrar aquí en debates terminológicos (Almansa Sánchez 2011; DíazAndreu 2016a, 2016b; Salerno 2012), la vertiente más social de la arqueología nace con la denominada "arqueología comunitaria"; un ensayo hacia la, por así llamarla, descolonización profesional de la ciencia arqueológica a través de la integración de miembros de las comunidades en las excavaciones de los espacios arqueológicos relacionados con su propia historia o la de sus ancestros (Marshall 2002). Teóricamente, esta forma de practicar arqueología, capacitando a la comunidad y no predicando sobre ella, fomentando una construcción del conocimiento proactiva y proyectiva, ha empezado a transformar la disciplina arqueológica
(Simpson 2009, 2010). Muchos de los proyectos de arqueología comunitaria surgen de un interés por conocer a fondo los valores sociales y educacionales asociados al pasado, su relación con el presente y con el público (Simpson y Williams 2008:69). En algunos casos, la arqueología comunitaria se ha confundido con la gestión de recursos culturales, o gestión de patrimonio en vez de considerarse como una práctica académica en sí misma (Little 2009:30), lo que ha conducido a reflexionar sobre los límites y aportaciones reales de la misma a la sociedad (Marshall 2002:213).

Pero ¿es la arqueología comunitaria una "arqueología desde abajo", contrapuesta a una "arqueología desde arriba" gestionada por las instituciones? (Faulkner 2000:22). En los contextos poscoloniales, y en realidad en todos los lugares, incluso los que no se han visto sujetos en algún momento de su historia al yugo del colonialismo (Apaydin 2018; Ayán Vila 2012), la arqueología comunitaria ha jugado un rol integrador, como una disciplina que establece un vínculo con el medio material y social que estudia (Atalay 2012; Byrne 2012; Carman 2011; Field et al. 2000; Matthews 2019; Van den Dries y Van der Linde 2012; Wright 2015). Pero algunos de estos trabajos se han podido configurar desde una "mentalidad gobernadora", extractivista, que prioriza el conocimiento de los expertos intelectuales que generan una arqueología participativa, que no comunitaria, sino más bien autoritaria. Nosotras creemos que la "autoridad" que ello representa puede ponerse a prueba y además dar lugar a prácticas que denoten un mayor aporte a la resolución de conflictos actuales:

La cultura material, o patrimonio, convierte a la cultura en un elemento esencial de la sociedad, en un conocimiento arqueológico tangible, "evidencial" y por lo tanto "real". Este carácter físico hace que los problemas sociales que interactúan con el patrimonio estén abiertos a una intervención y regulación (Smith 2004:10) (traducción de las autoras).

Según Smith, la cultura es un elemento fundamental de cualquier sociedad, y su materialidad, así como su conservación, puede ser objeto de conflicto social en diversos casos (Smith 2004:10). Virginia Salerno señala que:

es preeminente la presencia del conflicto en la articulación arqueologíasociedad [...] El conflicto que atraviesa 
estos problemas se deriva del lugar legitimado de los arqueólogos como agentes autorizados para llevar cabo ciertos procedimientos y construir conocimiento sobre el pasado" (Salerno 2013:15).

Si Salerno está en lo cierto, tendríamos que explorar ahora si esta faceta descolonizadora se puede extrapolar a contextos sociales en los que la comunidad se nutra de poder experimentar nuevos discursos patrimoniales no impuestos por los entes de poder, descolonizándose de las pautas de saber de la comunidad gobernante o experta. No es fácil llevar a cabo este tipo de proyectos, que pueden llegar a ser "extremadamente lentos, profundamente frustrantes, humillantes y desafiantes en formas imprevistas", pero como indica Marshall: "También es gratificante en formas que trascienden los estrechos elogios académicos" (Marshall 2002:2018).

La otra gran rama de la arqueología social es la arqueología pública -aunque se podría indicar que estas diferenciaciones atienden a una nube terminológica en torno a público, comunidad y participación (DíazAndreu 2016a:4). ¿Y qué es la arqueología pública? Jaime Almansa nos propone esta definición:

simplemente debemos entender la arqueología pública como un paraguas bajo el que suceden muchas cosas en común; entender cómo trabajamos y buscar estrategias para hacerlo mejor en el contexto político, social y económico en el que nos encontramos (Almansa Sánchez 2013:2).

A pesar de su diversidad, la arqueología pública se identifica, como su nombre indica, con un manifiesto interés en la participación del público ${ }^{1}$, pero no queda claro cuál es el mejor método para conocer al público, o de conocer de qué forma el público se relaciona con la arqueología o el patrimonio (Cardona 2016; Endere et al. 2018; Moshenska 2013, 2015; Richardson y Almansa Sánchez 2015).

La pregunta que cabe hacerse en este punto de nuestra reflexión es que, si la arqueología como una ciencia al servicio de la sociedad ha ido mutando en los últimos años a través de la integración de corrientes que abogan por cuestionarse la otredad o abordar la materialidad de forma multitemporal (p.ej., Criado Boado 2012; Gnecco 2013, 2017; González Ruibal
2012; Haber 2016; Hamilakis y Jones 2017; Olsen y Pétursdóttir 2014), ¿por qué la conservación no ha ido transformándose de forma pareja? ¿Por qué se habla de arqueología pública, comunitaria y no de conservación pública, comunitaria, o simplemente social?

La desvinculación de la conservación del debate sobre la arqueología pública o comunitaria puede deberse a diversas razones. Por una parte, podríamos lamentar que la arqueología más teórica no haya prestado atención, o incluso se haya desvinculado, de abordar cómo el público puede participar en la preservación del registro excavado. Por otra parte, lo cierto es que también la conservación arqueológica se ha centrado más en comunicar avances prácticos o ejecuciones de proyectos a nivel material, y no en reflexionar sobre cómo co-crear un conocimiento proyectivo en el marco arqueológico (Carrera Ramírez 2018). Esta falta de acercamiento teórico desde la conservación a lo social está de alguna manera asociada a una cuestión práctica: a una falta de diálogo entre técnicos arqueólogos y restauradores de arqueología, especialmente en las fases de planificación de las intervenciones (Barcia et al. 2016; Cortés-Díaz y Valtierra Pereiro 2018; Pastor Pérez 2016, Pastor Pérez et al. 2018).

La conservación arqueológica se ejecuta en muchos casos de forma descontextualizada, prestando únicamente atención a la recuperación de objetos, sin evaluar o valorar su entorno o contexto actual de forma holística. Una conservación que ponga en valor la multitemporalidad y usos o re-usos de los espacios arqueológicos será una conservación más social, analítica y crítica. Esta nueva conservación deberá ir acompañada de una evolución en la concepción de valor patrimonial, como veremos en la sección siguiente.

\section{Evolución de los Valores del Patrimonio}

¿Cómo podemos llegar a una conservación social? Desde el siglo XIX, época en la que los Estados comienzan a preservar el patrimonio arqueológico de la nación, la decisión sobre qué preservar ha recaído en manos de expertos (Choay 2001; DíazAndreu 2007; Jokilehto 1986). En 1903 se empleó por primera vez el concepto de valor para explicar por qué los monumentos y los sitios arqueológicos -entre otros- deberían conservarse (Riegl 1987 [1903]). Posteriormente, en los años setenta del siglo XX, la eclosión del nuevo escenario neoliberal necesitaría 
documentar y cuantificar la valía del objeto patrimonial arqueológico, con lo que el concepto de valor tomó una nueva forma, la económica-mercantilizada, esencial para moldear las decisiones de preservar o no un bien. Pero ¿quién decide qué posee o no posee valor? Como ya hemos comentado, la atribución de valor, valorización o puesta en valor del patrimonio, también el arqueológico, ha sido habitualmente la responsabilidad directa de los expertos y las administraciones públicas (Baxter 2010; Carman 2003; Darvill 2007; Stovel 2008; Swensen et al. 2013). Los valores se han ido tipificando para facilitar su uso a lo largo del tiempo, especialmente para condensar las formas en las que se pueden describir los bienes o conjuntos patrimoniales, para elaborar proyectos o discursos, así como evaluar el impacto de nuestras acciones en los bienes culturales como expertos (Labadi 2013:7).

Los valores evolucionan con el tiempo y con los cambios sociales, y han generado una gran cantidad de publicaciones en las últimas décadas, especialmente en los últimos 10 años (Armitage e Irons 2013; Burtenshaw 2014; Carver 1996, 2003; Cleere 1996; Díaz-Andreu 2016b; Fouseki et al. 2020; Francisco 2020, Fredheim y Khalaf 2016; Ives y Kendal 2014; Kajda et al. 2018; Labadi 2007; Lipe 1984; PargaDans y Alonso-González 2019; Pastor Pérez 2016, 2018, 2019; Pastor Pérez et al. 2018; Pastor Pérez y Ruiz Martínez 2016, 2018). Marta de la Torre, en sus múltiples estudios acerca de los valores del patrimonio (De la Torre 2002, 2013, 2014), nos propone una serie de características de estos: que son atribuidos, múltiples, mutables, inconmensurables y que a veces pueden entrar en conflicto (De la Torre 2013:159161). Citando a la autora, "cuando establecemos el significado de un lugar ponderamos más unos valores que otros, estableciendo así distintas pautas de conservación que provocarán que unos valores sobrepasen a otros, pudiendo quedar algunos en el olvido" (De la Torre 2013:159). Además, nos comenta que "no se pueden conservar todos los valores que se han atribuido a un sitio de forma simultánea, ya que algunos pueden ser contrarios a otros (conservación vs. uso simbólico)" (De la Torre 2013:159-161). A nuestro parecer, también, los valores se transforman con los cambios sociales, motivo por el cual han ido evolucionando y adaptándose a la deseada socialización del patrimonio que proponen las teorías críticas previamente comentadas. Como observamos en el resumen de la Tabla 1 inspirado en De la Torre (2013, 2014), no existen unos valores universales, sino que distintos profesionales han tipificado una serie de características para ellos desde principios del siglo XX (Riegl 1987 [1903]).

Los valores más mencionados entre la publicación de Riegl y la Carta de Burra de 1979 eran los que se asociaban a las características intrínsecas de los objetos: estético e histórico; un reconocimiento que le viene dado al objeto por haber pervivido al paso del tiempo o por representar una serie de cualidades técnicas que denotan la evolución del conocimiento humano en torno a la manufactura o artesanía. Como se puede observar, por tanto, la naturaleza de estos valores estaba completamente desvinculada del rol

Tabla 1. Resumen cronológico de los valores del patrimonio, inspirado en De la Torre (2013, 2014). Pastor Pérez (2018, 2019). Chronological summary of heritage values, inspired by De la Torre (2013, 2014). Pastor Pérez (2018, 2019).

\begin{tabular}{|c|c|c|c|c|c|}
\hline Alois Riegl (1903) & $\begin{array}{l}\text { ICOMOS Carta de } \\
\text { Burra (1979-2013) }\end{array}$ & $\begin{array}{c}\text { Bruno Frey / } \\
\text { Económicos } 1992\end{array}$ & English Heritage 1999 & $\begin{array}{l}\text { Cohen y Reguera/ } \\
\text { Contextuales } 2012\end{array}$ & $\begin{array}{c}\text { Barreiro y Criado "Cadena } \\
\text { de Valor" } 2015\end{array}$ \\
\hline Antigüedad & Estético & Prestigio & Estético & Estético & Estético \\
\hline Histórico & Histórico & & Histórico & & \\
\hline \multirow[t]{3}{*}{ Conmemorativo } & Nacional & Legado & Rareza & & De opción/ Legado \\
\hline & Científico & & Científico & & Documental \\
\hline & Social & & Social & Recreacional & \\
\hline \multirow[t]{2}{*}{$\begin{array}{l}\text { Novedad/ } \\
\text { Contemporaneidad }\end{array}$} & Cultural & & & Cultural & \\
\hline & & Existencia & Condición & & Existencia \\
\hline \multirow[t]{5}{*}{ Instrumental/ Uso } & & Opción & & Recurso & Instrumental \\
\hline & & Monetario & & Económico & Económico \\
\hline & & Educacional & & Educacional & Pedagógico \\
\hline & Político & & & & Político \\
\hline & Espiritual & & Espiritual & & \\
\hline
\end{tabular}


social de los objetos o conjuntos patrimoniales que, a partir de la Carta de Burra, se han comenzado a tener en cuenta. Volviendo a las características mencionadas por Marta de la Torre $(2013,2014)$, estas nos indican un giro hacia la dimensión social de estos valores, que toman impulso a raíz de los intentos de inclusión del público en los procesos de valorización (Clark 2006, 2009). Sin embargo, los propios expertos no parecen acabar de evaluar el espectro de sus acciones integradoras ni saben medir el impacto social de sus propuestas. En la introducción al volumen recopilatorio que publicó el Instituto de Conservación Getty (Los Ángeles, EUA), precisamente acerca de la evaluación de los valores del patrimonio cultural, De la Torre ya nos hablaba de la relación entre conservación y sociedad, indicando que:

Los grupos de agentes que representan los valores sociales suelen ser miembros del público que tradicionalmente no han participado en nuestro trabajo o cuyas opiniones no han sido tenidas en cuenta. Hoy en día, al reconocer la importancia de incluir a todos los agentes interesados en el proceso, debemos recurrir a otras disciplinas para que estos nuevos grupos participen en los debates (De la Torre 2002:3).

Los valores son, en muchos casos, utilizados como descriptores de los estudios de caso en los que trabajan los expertos, que se sirven de ellos en un intento de jerarquizar en las cualidades de un sitio. Así se establecen unas pautas de actuación de conservación que reflejen una serie de prioridades (habitualmente económicas) sustentadas por estos valores: nunca podremos conservar a la vez todos los valores que se atribuyen a un sitio, y menos aún, si se aborda el patrimonio como un recurso a explotar y no como un proceso cultural o una construcción social multivocal (Avrami 2009, 2011; Avrami et al. 2000). El análisis de la atribución y preservación de estos valores al patrimonio articula los discursos entre agentes, entre ellos los que acontecen entre los expertos y la sociedad. Una posibilidad de socializar estos valores sería entonces la de indagar en la dimensión no lineal de su legado; en la multitemporalidad de su misma existencia como fuente documenal (Barreiro Martínez y Criado Boado 2015).

Según David L. Uzzell, "Los lugares cambian a lo largo del tiempo. El significado de los lugares cambia a lo largo del tiempo" (Uzzell 1998:3) y quizás vivimos en un tiempo en que las vidas de los objetos se están acortando y la conservación juega un rol primordial en la pervivencia de las historias que se generan -si es que se desea que pervivanCada vez generamos más objetos perecederos, lo que resitúa la conservación como disciplina al servicio del entendimiento del pasado, a la vez que colabora con la desaparición de las líneas temporales (Millán Pascual 2015). En palabras del experto en historia del cine Thomas Elsaesser:

La obsolescencia es "historia estancada",
variando el famoso aforismo de Benjamin
sobre la imagen alegórica. Pero al detener
la historia, suspenderse el tiempo e
invertir su curso, la obsolescencia puede
suponer un momento de reevaluación y de
renovación, por lo que quiero insistir en
que la obsolescencia implica una relación
especial entre pasado y presente que ya
no sigue la linealidad directa de la causa
y el efecto, sino que toma la forma de un
bucle, en el que el presente redescubre
un pasado determinado, al que atribuye
entonces el poder de dar forma a aspectos
del futuro que ahora son nuestro presente
(Elsaesser 2015:113) (traducción de las
autoras).

En la era de la obsolescencia programada, de la estandarización postfordista, en que el registro arqueológico de Ikea guiará a los arqueólogos del futuro, la conservación social del patrimonio arqueológico tiene un rol indiscutible en la búsqueda de identidades multitemporales y multivocales que confieran una unicidad no estandarizada, con la óptica de una perspectiva histórica más extensa, que se extrapole a una espacial, palpable en los usos cotidianos (Armitage y Guldi 2015; Fowles 2016; Uzzell 1996:227; Uzzell et al. 2002), que vaya más allá de la idea de preservar para el goce de las generaciones futuras (ICOMOS Australia 2013 [1979]). En esta línea, las aproximaciones teóricas encaminadas a superar las tradicionales preguntas sobre "cómo", "dónde" y "qué" conservar y su sustitución por "por qué" conservar (y de qué modo extrapolarlo al eje de valor) serían el principal motor del giro ontológico en torno a la gestión de patrimonio de las últimas décadas (Harrison 2018a, 2018b:1636). En nuestra opinión, estos cambios deben entenderse 
como un llamamiento a abordar la conservación desde numerosas disciplinas como son la sociología, la geografía humana, la antropología... y con especial interés en que todas estas disciplinas converjan hacia un objetivo común: mitigar las relaciones de poder que se establecen de forma desigual entre autoridades, expertos y sociedad, y al servicio de las cuales han estado los valores del patrimonio y la disciplina de la conservación de bienes culturales arqueológicos (Barreiro y Varela-Pousa 2018; Harrison 2018b:1367; Pastor Pérez 2016, 2018).

\section{[De]cosificación y Des-elitización y del Patrimonio Arqueológico: Conservando los Contextos}

Si la conservación se rebelase contra la cosificación y elitización del patrimonio arqueológico, podría ejercer un papel socializador, ya que es una disciplina que aporta en sí misma narrativas sobre la historia de los objetos (Taylor 2015). Iniciaremos esta sección citando al arqueólogo gallego David Barreiro, quien, en su libro Arqueológicas. Hacia una Arqueología Aplicada, publicado en 2013, nos planteaba cuestiones como la siguiente:

\section{¿Puede contribuir la arqueología como práctica tecnocientífica a extender una conciencia patrimonial no cosificada y, por lo tanto, a configurar una racionalidad diferente, una racionalidad que anticipe y siente las bases de un modelo social y económico alternativo? (Barreiro Martínez 2013:63) $)^{2}$.}

Nos gustaría remarcar aquí que "[de]cosificar" el patrimonio arqueológico es una tarea compleja que debe comenzar durante el periodo de excavación, ya que, durante el mismo, el culto al objeto, su reificación y fetichización (Alonso González 2017), es habitual, y muchos directores y directoras de los trabajos arqueológicos en yacimientos siguen considerando los hallazgos como propios. Este sistema autárquico, muy frecuentemente "secuestra" la información obtenida -a veces durante años- a la espera de que vea la luz una publicación de impacto; un detalle que en muchos casos supone un riesgo de conservación en sí mismo, ya que muchas piezas pasan demasiado tiempo en almacenes con peores sistemas de conservación que los museos, y totalmente descontextualizadas ${ }^{3}$.

¿Cómo puede desvincularse un o una conservadorrestaurador/a de arqueología de ejercer esa reificación del objeto material? Un inicio, a nuestro parecer, sería generar estrategias de conservación contextual, es decir, que no afecten únicamente a los objetos sino a aquello que les circunda. y donde las transformaciones de los espacios y los usos actuales de los mismos jueguen un papel predominante en las intervenciones. En el año 2001 David Harvey exponía desde la óptica de la geografía una base teórica para el entendimiento de la "patrimonialización" como un proceso. Su texto es inspirador:

Cada sociedad ha tenido una relación con su pasado, incluso aquellos que han decidido ignorarlo, y es a través de la comprensión del significado y de la naturaleza de aquello que la gente se narra una a otra sobre su pasado, sobre lo que olvidan, recuerdan, memorizan o engañan, que los estudios de patrimonio podrán conectar con los debates académicos, más allá de los confines de la cultura centrada en el presente, divertimento, ocio y turismo (Harvey 2001:320) (traducción de las autoras).

Cuando Harvey nos indica que debemos entender el significado de "aquello que la gente se narra", ello puede percibirse también como una invitación a comprender el papel que juegan los restos arqueológicos patrimoniales, y sus discursos, en lo cotidiano. Harvey propone también "des-elitizar" el patrimonio y abrirlo hacia la memoria colectiva: aquí cita el trabajo de Pierre Nora (1989), indicando que "dibuja una distinción entre una élite, que posee una memoria institucionalizada y preservada en los archivos, y una memoria de la gente ordinaria, sin registrar, arraigada en las no mencionadas tradiciones y hábitos de la vida diaria" (Harvey 2001:36). El autor sugiere abordar aquello que sucede en todas las épocas y a todos los niveles:

Debemos dotar al patrimonio de una historia propia, no en términos de relatar la historia de la evolución de una determinada rama modernista del patrimonio a partir de un icono del siglo xix, sino en términos de examinar la evolución del proceso patrimonial a largo plazo (Harvey 2001:326).

En esa misma línea, el conservador Joel Taylor nos indica la idea de que: 
los sitios patrimoniales pueden haber sufrido grandes cambios a lo largo del tiempo, tanto físicos como interpretativos y esto tiene profundas implicaciones en la forma en que estos sitios se usan, preservan, interpretan y aprecian (Taylor 2015:75).

Este conservador, inspirado en parte por el Documento de Nara (ICOMOS 1994), remarca que parte del mensaje de esas transformaciones se transfiere a través de aquello que se ha conservado, por lo que el binomio conservación-contexto actual son fundamentales a la hora de abordar estrategias efectivas (Taylor 2015:76).

Aparte de las estrategias de conservación contextuales, desde este texto nos gustaría alentar también a caminar hacia una conservación arqueológica de los cuidados (Alarcón García 2007:234-236; Reading 2015), que explore las emociones que se generan en los procesos de conservación per se. $\mathrm{La}$ extrapolación de sentimientos de pertenencia elitistas y cosificadores del patrimonio arqueológico por parte de sus descubridores (y posteriormente sus gestores) es una acción completamente opuesta a los principios indicados en la Carta de Burra (ICOMOS Australia 2013 [1979]). Esta deriva habitualmente en una falta de diálogo y, a la larga, en deterioros irrecuperables o pérdidas materiales, que en este sentido no lo son únicamente para un equipo de investigadores concreto, sino para la sociedad en general. En estos casos, no se estaría produciendo una transferencia real del conocimiento para las generaciones del futuro, sino que el conocimiento quedaría secuestrado para el beneficio de unos pocos. La metodología arqueológica "tradicional" actual se convierte en una barrera en sí misma para integrarse en la evolución crítico-teórica del patrimonio cultural (en este caso arqueológico), incluso cuando las teorías posprocesuales invocaban a un cambio de mentalidad (Hodder 2005 [1992]), que no siempre se aplica a nivel de campo (Kristiansen 2014). En esa praxis no parecemos tener en cuenta la arqueología de los afectos y las emociones (Hamilakis 2013:201; Penrose 2013) que, en nuestra opinión, debería ser indispensable al abordar los procesos de patrimonialización. Como indica Sarah Tarlow:

Las arqueologías de la emoción y el afecto pueden contribuir a que las cosas, los lugares y la materialidad del mundo adquieran sentido emocional y ayuden a estructurar las relaciones emocionales entre las personas y las formas en que las personas se relacionan con su entorno (Elsaesser 2012:180).

¿Tienen en cuenta los conservadores de patrimonio arqueológico ese valor empático hacia la sociedad y los restos materiales? La disciplina de conservación arqueológica aún no está lo suficientemente conectada a la historiografía, la geografía humana, la antropología o la propia arqueología de campo (Edgeworth 2006) y es impensable encontrar en los informes algún párrafo que describa las emociones que siente el restaurador o restauradora al extraer o manipular un objeto. No existe aún una conciencia que integre o describa las emociones que los restos del pasado producenen los técnicos/as que los manipulan, que describa la resignificación de los artefactos o "cosas" en el momento de la extracción, que conecten presente y pasado, generando una transtemporalidad de los objetos (Armitage y Guldi 2015).

En el caso de los restauradores de arqueología, especialmente en España, la formación les "entrena" a seguir un camino pautado por otros, por los arqueólogos en el caso de la conservación arqueológica, en parte por su consideración de estudios no universitarios (Ruiz de Lacanal 2001; Viñas Lucas 2008). Tampoco suelen participar en la toma de decisiones previas a excavar y ejercen como mercenarios de salvaguarda mientras se produce la destrucción del registro, la transformación de un paisaje que configura la siguiente creación o [re]creación del registro arqueológico (Pastor Pérez y Canseco Domínguez 2016). La idea de acudir a un conservador de arqueología como último recurso, como recuperador de objetos perecederos, ha convergido hacia una realidad; una falta de contacto casi absoluta entre teoría arqueológica (y más aún social) y conservación. En oposición a esta línea de trabajo, lo que nosotras proponemos es que la conservación arqueológica, como integrante proactiva de esta tecnociencia, fomente que los espacios arqueológicos se conserven como sitios donde tienen lugar los procesos patrimoniales, y donde se produce un contacto y sinergia entre técnicos, patrimonio y habitantes. Creemos que la conservación arqueológica no se debe separar de las nuevas corrientes teóricas que abogan por la socialización de la arqueología, su multitemporalidad y su capacidad de respuesta a los problemas del presente (Almansa Sánchez 2017; González-Ruibal 2013; Hamilakis 2017). Para 
ello consideramos necesario que la conservación se integre en las corrientes sociales que se proponen desde disciplinas como la arqueología pública y comunitaria. En el siguiente apartado propondremos algunas reflexiones que sirvan como un punto de partida en la búsqueda de mitigar las distancias que existen tanto entre expertos (arqueólogos-conservadores) como entre estos y la sociedad.

\section{¿Hacia una Conservación Pública, Comunitaria, Social, Participativa?}

Las corrientes críticas en patrimonio instan a dar voz a las comunidades locales en la elaboración de los discursos patrimoniales y al modo en que estos se articulan con la sociedad. La concepción del patrimonio como un proceso cultural ha dado lugar a repensar los valores que se le aplican para su conservación, explorando la dimensión social de los mismos en detrimento de las nociones estéticas o históricas. Creemos que es momento de analizar, generar o repensar metodologías que tengan en cuenta tanto el tejido social como a todos los usuarios del espacio o del bien cultural en las definiciones de valor y autenticidad de los distintos discursos, rompiendo así con la exclusividad que hasta ahora han mantenido los y las expertos/as (Deacon y Smeets 2013:141).

Para que el rol de la comunidad o comunidades locales se respete y no se vea condicionado o apropiado por otros -en muchos casos la administración o fundaciones privadas-, además de ajustarse a lo que promueven los marcos legales actuales, hay que buscar sistemas que analicen e integren cuáles son los intereses de esta comunidad, evitando un desempoderamiento de la misma, pero también capacitándola para la identificación o mantenimiento de su patrimonio, lo que supone buscar encuentros simétricos entre agentes (Deacon y Smeets 2013:131; González-Ruibal et al. 2018; Waterton y Smith 2010). El estudio de los valores enraizados o basados en el tejido social y comunidades (Fusco Girard 2014; Niglio 2014) es clave para la socialización de la conservación de bienes culturales arqueológicos. Desde nuestro punto de vista, para resignificar la conservación de estos bienes, debemos comenzar por reexaminar los valores que se les atribuyen. Las encuestas realizadas para la tesis doctoral de una de las autoras de este trabajo denotan que el principal valor que los expertos atribuyen a los bienes arqueológicos es el histórico, basándose en criterios muy académicos (Pastor Pérez 2018:77, 2019) o autoritarios (Alonso González 2015:30).
Lo que aquí proponemos es que el valor histórico, de rasgo estético y estático, y el valor de legado, asociado a una época concreta, pueden transformarse en valores cinéticos, sociales e integradores. Pero para que ello suceda tendríamos que, por una parte, [de]cosificar y des-elitizar el patrimonio, y por otra, acudir a distintas disciplinas para entender cómo este interactúa realmente con la sociedad actual de su entorno (Armitage e Irons 2013; De la Torre 2002:3; Pastor Pérez y Ruiz Martínez 2018).

La arqueología es una ciencia que ha traspasado las fronteras temporales y se ha adentrado en conocer -e integrar- los aspectos contemporáneos del pasado en el presente, poniendo un especial énfasis en las vertientes más cotidianos de la vida humana. En este trabajo hemos intentado dirigirnos hacia la búsqueda de un espacio de interconexión teóricoconceptual necesario, que explore la dimensión social o democratizadora de la conservación arqueológica para su aplicación tanto en el campo como en el laboratorio. Este tipo de conservación, que propone una de-cosificación del patrimonio, busca desvincular la conservación arqueológica de la protección exclusiva y aislada del objeto y acercarla a la conservación de contextos espaciales atemporales (Kolar y Zabkar 2010; Pastor Pérez y Canseco Domínguez 2016:215; Pastor Pérez et al. 2018; Stovel 2008). Preservar el contexto conlleva también la supervivencia del carácter multitemporal de los restos, lo que nos puede ayudar a entender el rol que desempeña el objeto en la contemporaneidad, conservando así su proceso cultural per se, integrándose las nuevas corrientes críticas en patrimonio (Fouseki et al. 2019). Es por ello que reiteramos que la conservación debe adaptarse a las nociones de multitemporalidad, traspasando así la frontera de unos cuidados sobre el objeto que atiendan únicamente a los criterios de antigüedad y autenticidad. En este sentido, debe integrar los usos actuales de los espacios patrimoniales y el rol que estos juegan en la sociedad del presente. Citando al antropólogo Néstor García Canclini:

No se logrará una política efectiva de preservación y desarrollo del patrimonio si este no se valora adecuadamente por el público de los museos y los sitios arqueológicos [...] no basta multiplicar las investigaciones patrimoniales, los museos y la divulgación; hay que conocer y entender las pautas de percepción y comprensión en que se basa la relación con 
los destinatarios de los bienes culturales (García Canclini 1999:24).

Creemos que faltan metodologías que permitan comprender de una forma más compleja qué es lo que realmente entendemos por el público (tejido social o usuarios del espacio público) y de qué maneras los públicos diversos interactúan con el patrimonio arqueológico (Holtorf 2011; Murzyn-Kupisz y Działek 2013; Turner y Tomer 2013). En este sentido, lo que nos parece más necesario es, por una parte, invertir el tiempo suficiente en conocer hacia qué tipo de público se dirigen nuestras acciones, y por otra, cuáles son las expectativas y necesidad de este. Comprender cómo la sociedad se aproxima al patrimonio debe ser considerado un punto inicial previo a cualquier intervención, ya que sin él sería inviable confeccionar cualquier proyecto de conservación arqueológica, y menos aún aquellos que se identifiquen con un enfoque participativo-social. A la arqueología comunitaria y pública le hace falta un corpus teórico que vaya más allá de la multiplicidad metodológica que reflejan los abundantes estudios de caso que se publican (Gould 2016) y avanzar hacia cómo medir su impacto real en la sociedad, a corto y largo plazo (Guttormsen y Hedeager 2015). Además, en muchas ocasiones el carácter efímero de estos proyectos, supeditados a subvenciones puntuales, lega una obligación por y para la comunidad, que será la encargada de prolongar las acciones tanto divulgativas como de conservación; señalando un carácter usufructuario en contraposición a formativo (Salazar et al. 2020).

La arqueología y conservación comunitarias, públicas o participativas, tendrán que esperar a un cambio, o a la expansión de una arqueología más activista, como la que proponen a nivel internacional Sonia Atalay ${ }^{4}$ desde la UMass University, Jaime Almansa ${ }^{5}$ (CSIC-Incipit), el GAS $^{6}$ y el CRAS ${ }^{7}$ en España, Virginia M. Salerno (Salerno et al. 2016) desde el CONICET de Argentina y Henry Tantaleán o Miguel Aguilar (editores del libro Arqueología Social Latinoamericana: De la Teoría a la Praxis de 2013) desde Perú, entre otros. Esta arqueología activista, desde nuestro punto de vista necesaria, busca conectar y ayudar en los conflictos de las sociedades actuales, así como ejercer una cierta resistencia al mercantilismo existente a nivel de producción académica o del manejo de patrimonio como bien de consumo (Carrión et al. 2015). Estos cambios se adscriben a un contexto sociopolítico que no debe ser ignorado. En palabras del arqueólogo Nicolas Zorzin "el proceso de contextualización de la arqueología en la sociedad moderna constituye un acercamiento ético a la disciplina en sí mismo" (Zorzin 2015:116, traducción de las autoras). Ante este horizonte transdisciplinar y multitemporal, la preservación de los espacios arqueológicos debe transitar hacia su integración en los procesos patrimoniales, en las narrativas multivocales; debe convertirse en una conservación pública o colaborativa. En nuestra opinión, estamos a las puertas de un cambio en el cual la conservación arqueológica tendrá que volcarse hacia una transformación epistémica, de la misma forma que lo hicieron las corrientes críticas en arqueología y patrimonio, pero sin caer en actitudes extractivistas (Grosfoguel 2016).

Agradecimientos: A todas las compañeras y compañeros del Grup d'Arqueologia Pública i Patrimoni (GAPP) de la Universitat de Barcelona por estos años de intercambio de conocimiento, y los que vengan. Nos gustaría dar las gracias al comité editorial de la revista por su labor, y a los revisores anónimos por sus enriquecedoras sugerencias, que sin duda han ayudado a mejorar la calidad de este texto.

\section{Referencias Citadas}

Alarcón García, E. 2007. Las prácticas de los cuidados en las sociedades prehistóricas: la cultura Argárica. Arqueología y Territorio 4:233-249.

Almansa Sánchez, J. 2011. Arqueología para todos los públicos. Hacia una definición de la Arqueología pública "a la española". Arqueoweb: Revista sobre Arqueología en Internet 13 (1):87-107.

Almansa Sánchez, J. (ed.) 2013. Arqueología Pública en España. JAS Arqueología, Madrid.

Almansa Sánchez, J. 2017. Arqueología y Sociedad: Interacción y Acción desde la Teoría Crítica. Tesis doctoral, Facultad de Geografía e Historia, Universidad Complutense de Madrid, Madrid.

Alonso González, P. 2015. Conceptualizing Cultural Heritage as a Common. En Identity and Heritage. Contemporary Challenges in a Globalized World, editado por P.F. Biehl, D.C. Comer, C.
Prescott y H.A. Soderland, pp. 27-35. Springer International Publishing, Cham.

Alonso González, P. 2017. El Antipatrimonio: Fetichismo y Dominación en Maragatería. Consejo Superior de Investigaciones Científicas, Madrid.

Alonso González, P., D. González-Álvarez y J. Roura-Expósito 2018. ParticiPat: Exploring the Impact of Participatory Governance in the Heritage Field. PoLAR: Political and Legal Anthropology Review 41 (2):306-318.

Apaydin, V. 2018. Who knows what? Inclusivity versus exclusivity in the interactions of heritage and local-indigenous communities. En Shared Knowledge, Shared Power. Engaging Local and Indigenous Heritage, editado por V. Apaydin, pp. 29-44. Springer, Cham. 
Apaydin, V. (ed.) 2020. Critical Perspectives on Cultural Memory and Heritage Construction, Transformation and Destruction. UCL Press, London.

Armitage, D. y J. Guldi 2015. The Return of the Longue Durée: An Anglo-American Perspective. Annales (English Ed.) 70 (2):219-247.

Armitage, L. y J. Irons 2013. The values of built heritage. Property Management 31 (3):246-259.

Atalay, S. 2012. Community-Based Archaeology: Research With, By and For Indigenous and Local Communities. University of California Press, Berkerley.

Auclair, E. y G. Fairclough 2015. Theory and Practice in Heritage and Sustainability: Between Past and Future. Routledge, London.

Avrami, E. 2009. Heritage, Values and Sustainability. En Conservation: Principles, Dilemmas, and Uncomfortable Truths, editado por A. Richmond y A. Bracker, pp. 177-183. Butterworth-Heinemann, Oxford.

Avrami, E. 2011. Sustainability and Built Environment: forging a role for Heritage Conservation. Conservation Perspectives. The GCI Newsletter 26 (1):4-9.

Avrami, E., R. Mason y M. De la Torre (eds.) 2000. Values and Heritage Conservation. Research Report. The Getty Conservation Institute, Los Angeles.

Ayán Vila, X.M. 2012. Public archaeology, democracy and community. En Integrating Archaeology. Science-Wish-Reality. Social Role, Possinilities and Perspectives of Calssical Studies. International Conference on the Social Role, Possibilities and Perspectives of Classical Studies. Frankfurt 12-14 June 2012, editado por N. Schüker, pp. 41-47. Römisch-Germanische Kommission, Frankfurt.

Barcia, C., A. Pastor Pérez, A. Ortiz y A. Bofill 2016. Conservació i Restauració del Dolmen de la Taula dels Tres Pagesos (Forallac, Girona). Actas XIV Jornades d'Arqueologia de Les Comarques de Girona. Banyoles 11-13 Juny, pp. 77-84. Departament de Cultura, Generalitat de Catalunya.

Barreiro Martínez, D. 2013. Arqueológicas. Hacia una Arqueología Aplicada. Bellatera, Barcelona.

Barreiro Martínez, D. y F. Criado Boado 2015. Analizando el valor social de Altamira. Revista PH 87:108-127.

Barreiro, D. y R. Varela-Pousa 2018. Hacia una gestión de riesgos crítica en patrimonio cultural (18 de abril 2018). Actas del III Congreso de Buenas Prácticas de Patrimonio Mundial, 2-5 de mayo, Mahón, Menorca, editado por A. Castillo Mena. https:// eprints.ucm.es/id/eprint/55524/2/BPPM18-final.pdf.

Baxter, I. 2010. Building value through data. Heritage \& Society 3 (1)33-50.

Burtenshaw, P. 2014. Mind the gap: Cultural and economic values in archaeology. Public Archaeology 13 (1-3):48-58.

Byrne, S. 2012. Community archaeology as knowledge management: Reflections from Uneapa Island, Papua New Guinea. Public Archaeology 11 (1):26-52.

Cardona Gómez, G. 2016. Implicación social y patrimonio. Un cruce de caminos entre arqueología pública, arqueología comunitaria y didáctica de la arqueología. En Arqueología y Comunidad. El Valor del Patrimonio Arqueológico en el Siglo XXI, editado por M. Díaz-Andreu, A. Pastor Pérez y A. Ruiz Martínez, pp. 13-33. JAS Arqueología, Madrid.

Carman, J. 2003. Archaeology and Heritage: An Introduction. Bloomsbury Publishing, London.

Carman, J. 2011. Stories we tell: Myths at the heart of 'community archaeology'. Archaeologies 7 (3):490-501.

Carrera Ramírez, F. 2018. Conservación Preventiva en yacimientos arqueológicos: ¿empezamos? Actas del VI Congreso GE-IIC. ¿Y después? Control y Mantenimiento del Patrimonio Cultural, una Opción Sostenible. Vitoria. 20-22 septiembre 2018, pp. 376-384. Grupo Español IIC, Vitoria.

Carrión, H., C. Dávila, A. Delgado, N. Fuenzalida, P. Kelly, F. Moya, S. Rebolledo, S. Sierralta, J. Sepúlveda y C. González 2015. Evaluación de la Arqueología Social en Chile: desarrollo histórico y revisión crítica del proyecto disciplinar. Boletín de la Sociedad Chilena de Arqueología 45: 95-114.

Carver, M. 1996. On archaeological value. Antiquity 70 (267):45-56.

Carver, M. 2003. Archaeological Value and Evaluation (SAPManu). Società Archeologica Padana, Mantova.

Choay, F. 2001. The Invention of the Historic Monument. Cambridge University Press, Cambridge.

Clark, K. (ed.) 2006. Capturing the Public Value of Heritage. The Proceedings of the London Conference. English Heritage, London.

Clark, K. 2009. Values in cultural resource. Management. En Heritage Values in Contemporary Society, editado por G. Smith, P. Messenger y H. Soderland, pp. 89-99. Left Coast Press, Walnut Creek.

Cleere, H. 1996. The concept of 'outstanding universal value' in the World Heritage Convention. Conservation and Management of Archaeological Sites 1 (4):227-233.

Cortés-Díaz, A. y N. Valtierra Pereiro 2018. Presencia de conservadores-restauradores en yacimientos arqueológicos de España. En Las Profesiones del Patrimonio Cultural. Competencias, Formación y Transferencia del Conocimiento: Reflexiones y Retos en el Año Europeo del Patrimonio Cultural 2018, editado por A. Galán Pérez (ACRE) y D. Pardo San Gil (GE-IIC), pp. 68-74. Ministerio de Cultura y Deporte, Madrid.

Criado Boado, F. 2012. Arqueológicas: La Razón Perdida: la Construcción de la Inteligencia Arqueológica. Bellatera, Barcelona.

Darvill, T. 2007. Research Frameworks for World Heritage Sites and the Conceptualization of Archaeological Knowledge. World Archaeology 39 (3):436-457.

De la Torre, M. (ed.) 2002. Assessing the Values of Cultural Heritage. Getty Conservation Institute, Los Angeles.

De la Torre, M. 2013. Values and Heritage Conservation. Heritage \& Society 6 (2):155-166.

Deacon, H. y R. Smeets 2013. Authenticity, value and community involvement in heritage management under the world heritage and intangible heritage conventions. Heritage \& Society 6 (2):129-143.

De la Torre, M. 2014. Values in heritage conservation: A Project of The Getty Conservation Institute. APT Bulletin 45 (2/3):19-24. 
Díaz-Andreu, M. 2007. A World History of Nineteenth-Century Archaeology. Nationalism, Colonialism and the Past. Oxford University Press, Oxford.

Díaz-Andreu, M. 2016a. Introducción. En Arqueología y Comunidad. El Valor del Patrimonio Arqueológico en el Siglo XXI, editado por M. Díaz-Andreu, A. Pastor Pérez, y A. Ruiz Martínez, pp. 1-11. JAS Arqueología, Madrid.

Díaz-Andreu, M. 2016b. Arqueología, comunidad y valor social: un reto para el patrimonio arqueológico del siglo XXI. En Arqueología y Comunidad. El Valor del Patrimonio Arqueológico en el Siglo XXI, editado por M. Díaz-Andreu, A. Pastor Pérez, y A. Ruiz Martínez, pp. 69-90. JAS Arqueología, Madrid.

Edgeworth, M. (ed.) 2006. Ethnographies of Archaeological Practice. AltaMira, Lanham.

Elsaesser, T. 2015. Media archaeology as the poetics of obsolescence. At the Borders of (film) History Temporality, Archaeology, Theories: XXI Convegno Internazionale di Studi sul cinema $=$ XXI International film Studies Conference, 2014, editado por A. Beltrame, G. Fidotta, y A. Mariani, pp. 103-116. Forum, Udine.

Endere, M.L., M.G. Chaparro y M.E. Conforti 2018. Making cultural heritage significant for the public. The role of researchers in encouraging public awareness and local pride. Public Archaeology 17 (1):36-54.

Faulkner, N. 2000. Archaeology from below. Journal of Public Archaeology 1:22-33.

Field, J., J. Barker, R. Barker, E. Coffey, L. Coffey, E. Crawford, L. Darcy, T. Fields, G. Lord, B. Steadman y S. Colley 2000. 'Coming back' Aborigines and archaeologists at Cuddie Springs. Public Archaeology 1 (1):35-48.

Fiske, A.P. 1992. The four elementary forms of sociality: Framework for a unified theory of social relations. Psychological Review 99 (4):689-723.

Fowles, S. 2016. The perfect subject (postcolonial object studies) Journal of Material Culture 21 (1):9-27.

Francisco, J.P. 2020. Os valores do patrimonio: uma investigação sobre os sitios pré-históricos de arte rupestre do Vale do Rio Côa e de Siega Verde. Al-Madan 23 (1):99-113.

Fredheim, L.H. y M. Khalaf 2016. The significance of values: heritage value typologies re-examined. International Journal of Heritage Studies 22 (6):466-481.

Fouseki, K., T. Guttormsen y G. Swensen 2019. Heritage and Sustainable Urban Transformations: Deep Cities. En Heritage and Sustainable Urban Transformations: Deep Cities, editado por K. Fouseki, T. Guttormsen y G. Swensen, pp. 1-15, Routledge, London, New York.

Fouseki, K., J. Taylor, M. Díaz-Andreu, S.J. Van der Linde y A.R. Pereira-Roders 2020. Locating heritage value. En Locating Value: Theory, Application and Critique, editado por G. Hoskins y S. Saville, pp. 37-50. Routledge, London.

Fusco Girard, L. 2014. Towards the heritage community. ICOMOS International Symposium. Heritage and Landscape as Human Values. Firenze, Italia, 4-9 november, 2014, editado por M. Di Stefano pp. 410-411. ICOMOS, Firenze.
García Canclini, N. 1999. Los usos sociales del Patrimonio Cultural. En Patrimonio Etnológico: Nuevas Perspectivas de Estudio, editado por E. Aguilar Criado, pp. 16-33. Instituto Andaluz del Patrimonio Histórico, Junta de Andalucía.

Gnecco, C. 2013. Digging alternative archaeologies. En Reclaiming Archaeology: Beyond the Tropes of Modernity, editado por A. González-Ruibal, pp. 67-78. Routledge, Abingdon.

Gnecco, C. 2017. Antidecálogo: Diez Ensayos (Casi)Arqueológicos. JAS Arqueología, Ediciones del Signo, Popayán, Madrid, Buenos Aires.

González-Ruibal, A. 2012. Cada vez más islas. Arqueogazte. Aldizkaria 2:17-19.

González-Ruibal, A. 2013. Reclaiming Archaeology: Beyond the Tropes of Modernity. Routledge, Abingdon.

González-Ruibal, A., P.A. González y F. Criado-Boado 2018 Against reactionary populism: Towards a new public archaeology. Antiquity 92:507-515.

Gould, P. 2016. On the case: Method in public and community archaeology. Public Archaeology 15 (1):1-18.

Grosfoguel, R. 2016. Del extractivismo económico al extractivismo epistémico y ontológico. Revista Internacional de Comunicación y Desarrollo (RICD) 1 (4):33-45

Guttormsen, T.S. y L. Hedeager 2015. Introduction: interactions of archaeology and the public. World Archaeology 47 (2):189-193

Haber, A. 2016. Decolonizing archaeological thought in South America. Annual Review of Anthropology 45 (1):469-485.

Haber, A. 2019. Comentario a "en contra del populismo reaccionario: Hacia una nueva arqueología pública", de Alfredo González Ruibal, Pablo Alonso González y Felipe Criado-Boado. Chungara Revista de Antropología Chilena 51 (1):137-139.

Hamilakis, Y. 2013. Archaeology and the Senses: Human Experience, Memory, and Affect. Cambridge University Press, New York.

Hamilakis, Y. 2017. Sensorial assemblages: Affect, memory and temporality in assemblage thinking. Cambridge Archaeological Journal 27 (1):169-182

Hamilakis, Y. y A.M. Jones 2017. Archaeology and assemblage. Cambridge Archaeological Journal 27 (1):77-84

Harrison, R. 2013. Heritage. Critical Approaches. Routledge, Abingdon, New York.

Harrison, R. 2018a. Critical heritage studies beyond epistemic popularism. Antiquity 92 (365):e9. DOI: https://doi.org/10.15184/ aqy.2018.223.

Harrison, R. 2018b. On heritage ontologies: Rethinking the material worlds of heritage. Anthropological Quarterly 91 (4):1365-1384

Harvey, D. 2001. Heritage Pasts and Heritage Presents: temporality, meaning and the scope of heritage studies. International Journal of Heritage Studies 7 (4):319-338.

Hodder, I. 2005 [1992]. Theory and Practice in Archaeology. Routledge, London, New York.

Holtorf, C. 2011. The changing contribution of cultural heritage to society. Museum International 63 (1-2):8-16

ICOMOS 1994. The Nara Document on Authenticity. ICOMOS, Paris.

ICOMOS Australia 2013 [1979]. The Burra Charter: The Australia ICOMOS Charter for Places of Cultural Significance. ICOMOS, Australia. 
Ireland, T. y J. Schofield (eds.) 2015. The Ethics of Cultural Heritage. Springer, New York.

Ives, C.D. y D. Kendal 2014. The role of social values in the management of ecological systems. Journal of Environmental Management 144C:67-72.

Jokilehto, J. 1986. A History of Architectural Conservation. Ph.D. Thesis, Institute of Advanced Architectural Studies, Univeristy of York, New York.

Kajda, K., A. Marx y H. Wright 2018. Archaeology, heritage, and social value: Public perspectives on european archaeology. European Journal of Archaeology 21 (1):96-117.

Kolar, T. y V. Zabkar 2010. A consumer-based model of authenticity: An oxymoron or the foundation of cultural heritage marketing? Tourism Management 31 (5):652-664.

Kristiansen, K. 2014. Towards a new paradigm? The third science revolution and its possible consequences in archaeology. Current Swedish Archaeology 22:11-71.

Labadi, S. 2007. Representations of the nation and cultural diversity in discourses on World Heritage. Journal of Social Archaeology 7 (2):147-170.

Labadi, S. 2013. UNESCO, Cultural Heritage and Outstanding Universal Value. Altamira Press, Lanaham.

Lipe, W. 1984. Value and meaning in cultural resources. En Approaches to the Archaeological Heritage: A Comparative Study of World Cultural Resource Management Systems, editado por H. Cleere pp. 1-11. Cambridge University Press, Cambridge.

Little, B.J. 2009. Public archaeology in the United States in the early twenty-first century. En Heritage Studies. Methods and Approaches, editado por M.L. Sørensen y J. Carman, pp. 29-51. Routledge, London.

Londoño, W. 2014. Más allá del patrimonio. En Multivocalidad y Activaciones Patrimoniales en Arqueología: Perspectivas desde Sudamérica, editado por M.C. Rivolta, M. Montenegro, L. Menezes Ferreira y J. Nastri, pp. 155-167. Fundación de Historia Natural Félix de Azara, Facultad de Ciencias Sociales, Universidad Nacional de la Provincia de Buenos Aires, Buenos Aires.

Lowenthal, D. 1998. The Heritage Crusade and the Spoils of History. Cambridge University Press, Cambridge.

Lowenthal, D. 2014 [1985]. The Past is a Foreign CountryRevisited. Cambridge University Press, Cambridge.

Marshall, Y. 2002. What is community archaeology? World Archaeology 34 (2):211-219.

Matthews, C.N. 2019. Assemblages, routines, and social justice research in community archaeology. Journal of Community Archaeology \& Heritage 6 (3):220-226.

Millán Pascual, R. 2015. La desactivación de la protesta. Arqueología, conflicto, malestar. Arqueogazte 5:35-49.

Molina, B. 2019. Foros híbridos, participación y gestión sostenible del Patrimonio Mundial. El caso de Santa Ana de Cuenca. Methodos Revista de Ciencias Sociales 7 (2):225-243.

Moshenska, G. 2013. The archaeological gaze. En Reclaiming Archaeology: Beyond the Tropes of Modernity, editado por A. González-Ruibal, pp. 211-219. Routledge, Abingdon, Oxon, New York.
Moshenska, G. 2015. The ethics of public engagement in the archaeology of modern conflict. En Ethics and the Archaeology of Violence, editado por A. González-Ruibal y G. Moshenska, pp. 167-180. Springer, New York.

Murzyn-Kupisz, M. y J. Działek 2013. Cultural heritage in building and enhancing social capital. Journal of Cultural Heritage Management and Sustainable Development 3 (1):35-54.

Niglio, O. 2014. Inheritance and identity of cultural heritage. Advances in Literary Study 2 (1):1-4.

Nora, P. 1989. Between memory and history: Les lieux de memoire. Representations 26:7-24.

Oldham, M. 2017. Bridging the gap: Classification, theory and practice in public archaeology. Public Archaeology 16 (3-4):214-229.

Olsen, B. y P. Pétursdóttir (eds.) 2014. Ruin Memories: Materialities, Aesthetics and the Archaeology of the Recent Past. Routledge, Abingdon.

Parga-Dans, E. y P. Alonso-González 2019. Sustainable tourism and social value at world heritage sites: Towards a conservation plan for Altamira, Spain. Annals of Tourism Research 74:68-80.

Pastor Pérez, A. 2016. Towards a social archaeological conservation in Barcelona. Complutum 27 (2):259-280.

Pastor Pérez, A. 2018. Shaping community heritage synergies between Roman Barcelona spaces and the gothic neighborhood. En Shared Knowledge, Shared Power. Engaging Local and Indigenous Heritage, editado por V. Apaydin, pp. 61-86. Springer, Cham.

Pastor Pérez, A. 2019. Conservación Arqueológica Social. Etnografías Patrimoniales en el Barri Gòtic de Barcelona. Tesis de doctorado, Departament de Prehistòria i Arqueología, Universitat de Barcelona, Barcelonas.

Pastor Pérez, A. y O. Canseco Domínguez 2016. Hacia la autosostenibilidad en procesos de excavación: conservación preventiva y gestión de riesgos. Revista Otarq 1 (1):187216.

Pastor Pérez, A. y A. Ruiz Martínez 2016. Nuevas metodologías para una comprensión de las interacciones entre el público y el patrimonio arqueológico urbano. En Arqueología y Comunidad. El Valor del Patrimonio Arqueológico en el Siglo XXI, editado por M. Díaz-Andreu, A. Pastor Pérez y A. Ruiz Martínez, pp. 91-112. JAS Arqueología, Madrid.

Pastor Pérez, A. y A. Ruiz Martínez 2018. Analysing Heritage and Participation in the Gothic Quarter of Barcelona: Some methodological insights. Cuadernos de Prehistoria y Arqueología de la Universidad de Granada 28:115-147.

Pastor Pérez, A. y A. Ruiz Martínez 2020. ¿Somos el discurso académico autorizado patrimonial? En 21 Ensayos sobre el Patrimonio Cultural, editado por A. Pastor Pérez, M. Picas y A. Ruiz Martínez, pp. 63-67. JAS Arqueología, Madrid.

Pastor Pérez, A., A. Serrano Ramos, A. Monsalve Romera y M. Arco Hontoria 2018. Nuevos retos para la conservación preventiva en arqueología. El Cerro Bilanero (Ciudad Real, España). Revista Otarq: Otras Arqueologías 3:199-224.

Penrose, S. 2013. Archaeologies of the postindustrial body. En The Oxford Handbook of the Archaeology of the Contemporary World, editado por P. Graves-Brown, R. Harrison y A. Piccini, pp. 685-698. Oxford University Press, Oxford. 
Prats, L. 2012. El patrimonio en tiempos de crisis. Revista Andaluza de Antropología 2 (1):68-85.

Querol, M.A. 2010. Manual de Gestión del Patrimonio Cultural. Akal, Madrid.

Querol Fernández, M.A. 2011. El patrimonio cultural en las universidades españolas: no sólo cuestión de tiempo. Patrimonio Cultural de España 5:7589.

Quintero Morón, V. y C. Sánchez Carretero 2017. Los verbos de la participación social y sus conjugaciones. Contradicciones de un patrimonio "democratizador". Revista Andaluza de Antropología 12:48-69.

Reading, A. 2015. Making feminist heritage work: Gender and heritage. En The Palgrave Handbook of Contemporary Heritage Research, editado por E. Waterton y S. Watson, pp. 397-413. Palgrave Mcmillan, Basingstoke.

Richardson, L.J. y J. Almansa-Sánchez 2015. Do you even know what public archaeology is? Trends, theory, practice, ethics. World Archaeology 47 (2):194-211.

Riegl, A. 1987 [1903]. El Culto Moderno a los Monumentos: Caracteres y Origen. Machado Libros, Madrid.

Rivolta, M.C., M. Montenegro, L. Menezes Ferreira y J. Nastri (eds.) 2014. Multivocalidad y Activaciones Patrimoniales en Arqueología. Fundación de Historia Natural Félix de Azara Facultad de Ciencias Sociales. Universidad Nacional de la Provincia. de Buenos Aires, Buenos Aires.

Ruiz de Lacanal, M.D. 2001. El patrimonio cultural y su conservación en el sistema de enseñanza actual: una didáctica específica del siglo XXI. Las Didácticas de las Áreas Curriculares en el Siglo XXI: I Congreso Nacional de Didácticas Específicas. Granada, 1, 2 y 3 de febrero de 2001, editado por F.J. Perales Palacios, pp. 445-454. Grupo Editorial Universitario, Granada.

Salazar, D., J. Urrea-Navarrete, M. Escobar, P. Andrade, M. Muñoz, C. Maldonado, R. Torres y K. Vargas 2020. Arqueología y educación formal en la costa de Taltal-Paposo: reflexiones sobre "arqueología pública". Chungara Revista de Antropología Chilena 52 (4):651-669.

Salerno, V.M. 2012. Pensar la arqueología desde el sur. Complutum 23 (2): 191-203.

Salerno, V.M. 2013. Arqueología pública: reflexiones sobre la construcción de un objeto de estudio. Revista Chilena de Antropología 27 :7-37.

Salerno, V.M., M.C. Picoy, M. Tello, H.C. Pinochet, C. Lavecchia y G. Moscovici Vernieri 2016. Lo "público" en la arqueología argentina. Chungara Revista de Antropología Chilena 48 (3):397-408

Schofield, J. 2009. Being autocentric: Towards symmetry in heritage management practices. En Valuing Historic Environments, editado por L. Gibson y J. Pendlebury, pp. 93-114. Ashgate Publishing Company, Surrey.

Simpson, F. 2010. The Values of Community Archaeology: A Comparative Assessment Between the UK and US. BAR 2105 British Archaeological Reports, Oxford.

Simpson, F. 2009. Evaluating the value of community archaeology: The X Arch Project. Treballs d'Arqueologia 15:51-62.
Simpson, F. y H. Williams 2008. Evaluating Community Archaeology in the UK. Public Archaeology 7 (2):69-90.

Smith, L. 2004. Archaeological Theory and the Politics of Cultural Heritage. Routledge, London, New York.

Smith, L. 2006. Uses of Heritage. Routledge, London, New York.

Smith, R.C. y O.S. Iversen 2014. Participatory heritage innovation: Designing dialogic sites of engagement. Digital Creativity 25 (3):255-268

Sørensen, M.L.S. y J. Carman (eds.) 2009. Heritage Studies: Methods and Approaches. Routledge, Oxon.

Stovel, H. 2008. Origins and influence of the Nara Document on authenticity. APT Bulletin 39:9-17.

Swensen, G., G.B. Jerpåsen, O. Sætery y M.S. Tveit 2013. Capturing the intangible and tangible aspects of heritage: Personal versus official perspectives in cultural heritage management. Landscape Research 38 (2):203-221.

Tantaleán, H. y M. Aguilar (eds.) 2013. Arqueología Social Latinoamericana. De la Teoría a la Praxis. Universidad de los Andes, Bogotá.

Tarlow, S. 2012. The archaeology of emotion and affect. Annual Review of Anthropology 41 (1):169-185

Taylor, J. 2015. Embodiment unbound: Moving beyond divisions in the understanding and practice of heritage conservation. Studies in Conservation 60 (1):65-77.

Turner, M. y T. Tomer 2013. Community participation and the tangible and intangible values of urban heritage. Heritage \& Society 6 (2):185-198.

Uzzell, D.L. 1996. Creating place identity through heritage interpretation. International Journal of Heritage Studies 1 (4):219-228.

Uzzell, D.L. 1998. Interpreting our heritage: A theoretical interpretation. En Contemporary Issues in Heritage and Environmental Interpretation: Problems and Prospects, editado por D.L. Uzzell y R. Ballantyne, pp. 11-25. The Stationery Office, London.

Uzzell, D., E. Pol y D. Badenas 2002. Place identification, social cohesion, and environmental sustainability. Environment and Behavior 34 (1):26-53.

Van den Dries, M.H. y S.J. Van der Linde 2012. Collecting oral histories for the prupose of stimulating community involvement at Tell Balata (Palestine). En Integrating Archaeology. ScienceWish-Reality. Social Role, Possibilities and Perspectives of Classical Studies. International Conference on the Social Role, Possibilities and Perspectives of Classical Studies. Frankfurst 12-14 June 2012, editado por N. Schücker, pp. 49-56. RömischGermanische Kommission, Frankfurt.

Viñas Lucas, R. 2008. La conservación y restauración de bienes culturales en el nuevo contexto educativo español. PH: Boletín Del Instituto Andaluz del Patrimonio Histórico 66:106-123.

Waterton, E. 2015. Heritage and Community Engagement. En The Ethics of Cultural Heritage, editado por T. Ireland y J. Schofield, pp. 53-67. Springer, New York. 
Waterton, E. y L. Smith 2010. The recognition and misrecognition of community heritage. International Journal of Heritage Studies 16 (1-2):4-15.

Waterton, E. y S. Watson 2013. Framing theory: Towards a critical imagination in heritage studies. International Journal of Heritage Studies 19 (6):546-561.
Wright, A.P. 2015. Private property, public archaeology: Resident communities as stakeholders in American archaeology. World Archaeology 47 (2):212-224.

Zorzin, N. 2015. Archaeology and capitalism: Successful relationship or economic and ethical alienation? En Ethics and Archaeological Praxis, editado por C. Gnecco y D. Lippert, pp. 115-139. Springer, New York.

\section{Notas}

1 Claro está que lo que ahora se entiende como arqueología pública es muy diferente a como se definía en los años setenta (véase Díaz-Andreu 2016b:75-77).

2 En el original también aparece en cursiva.

3 La arqueología programada ha sido durante muchos años un espacio puramente académico, donde a los conservadoresrestauradores se les consultaba e integraba en el proyecto una vez que empezaban a salir vestigios, o incluso años después, cuando estos comenzaban a deteriorarse, dejando a la figura del conservador casi como la de un consultor externo, sin presencia en las etapas de planificación.

4 En su blog, la autora nos presenta un manifiesto para descolonizar la arqueología y los métodos: https://blogs. umass.edu/satalay/imagining-decolonial-research-futures/ (acceso 06/04/2019). Enlace al cómic Journeys to Complete the Work: https://blogs.umass.edu/satalay/repatriation-comic/ (acceso 06/04/2019).

5 El experto Jaime Almansa, en un monólogo titulado "Desmontando la imagen de la arqueología": https://tdjp. es/ensayo-fallido/ (acceso 06/04/2019).

6 Enlace al sitio web del Grupo de Arqueología Social (GAS) https:/grupoarqueologiasocial.wordpress.com/tag/gas/ (acceso 06/04/2019).

7 Enlace al sitio web del Centro Revolucionario de Arqueología Social (CRAS). http://www.arqueologiasocial.com/ (acceso 06/04/2019). 